\title{
Multipath Routing Protocol for DAG-based WSNs with Mobile Sinks
}

\author{
Gen $\mathrm{Xu}$ \\ Dept. of Computer Science and Technology \\ East China Normal University \\ Shanghai, China \\ e-mail: trueman1990@gmail.com
}

\author{
Gang Lu \\ Dept. of Computer Science and Technology \\ East China Normal University \\ Shanghai, China \\ e-mail: glu@cs.ecnu.edu.cn
}

\begin{abstract}
Low power and lossy networks have been an active area of research. Numerous protocols have been proposed for routing in these networks using metrics like hop count, delay, bandwidth etc. The IETF ROLL group has proposed a proactive gradient protocol called RPL. However, for a network with few mobile sinks calculating gradients using proactive approach is not energy efficiency. This paper proposed a multipath routing protocol for Wireless Sensor Networks with mobile sinks, called MDMR. Directed Acyclic Graphs (DAGs) are required for WSNs organization because of their route redundancy to the root. MDMR is based on RPL framework. By broadcasting DAG construction messages including metrics, such as, hop count, node energy and link quality indication, protocol constructs DAG and provides path redundancy. This allows nodes to find multiple alternative paths easily while link failures.
\end{abstract}

Keywords-WSN; Routing; DAG; RPL.

\section{INTRODUCTION}

Wireless Sensor Networks consist of hundreds of distributed sensors connected to each other. Today, sensors hold a wide area of usage such as in industry, environmental monitoring, healthcare applications and military applications. Yet, some challenges still remain to be overcome before this vision becomes a practical reality. One of them is that energy of nodes is quickly depleted since there are holes near the sinks [1]. Many techniques have been proposed to solve the problem. One way is to have mobile sinks.

Up to present, the routing strategy is a key crucial issue in WSNs with mobile sinks for links may frequently fails in some strict environment and can result in the network topology changing and failure of transferring data. Complicated techniques are needed to set up and maintain reliable paths, as well as detect link failures. However, existing routing algorithms, such as AODV and OLSR, are not suitable for these WSNs [2].

The Routing Over Low-power and Lossy Networks (ROLL) Working Group formed by the Internet Engineering Task Force (IETF) designs a new routing protocol called RPL (Routing Protocol for Low-power and Lossy Networks). The ROLL Working Group recently published the RPL as an RFC, named RPL IPv6 Routing Protocol for Low-Power and Lossy Networks [3]. As stated in the RFC,
RPL adopts a gradient routing strategy that organizes the WSN as a Directed Acyclic Graph (DAG). Each node selects at most three parent nodes. It considers the best parent node as the preferred one and uses it for packet forwarding to the border router. Alternate parents provide backup routes to the border router making the network robust to unexpected changes in radio connectivity (See Section II for details). However, the network structure built by RPL is different from the topology required in multi-hop networks with the 802.15.4 MAC layer-a cluster-tree. This latter topology allows a hierarchical organization in which a node can only select and associate with just one parent node [4].

In this paper, a multipath routing protocol for DAGbased WSNs with mobile sinks has been presented that based on the RPL framework. Although the framework has been specified in [3], there are still several open research problems not addressed by the RFC specification. For example, one of the most important issues is the behavior of objective functions. Unlike RPL, MDMR is based on a reactive on-demand approach, in that way, a node requests the sink to build a destination-oriented DAG (DODAG) to establish path between them. We use three routing metrics: hop count, node energy and link quality indication. In our paper, we combine the metrics in a lexical manner to compute rank. So the protocol can avoid loops by using a rank-based link selection process. Furthermore, the protocol can select the most powerful node and the best quality link.

The rest of paper is organized as follows: Section II provides an overview on the RPL protocol. Section III describes the proposed protocol in detail. Section IV reports and explains simulation results. Finally, the last Section draws conclusion.

\section{RELATED WORKS}

\section{A. Protocol overview}

IETF ROLL work group develop the RPL routing protocol especially for Low Power and Lossy Networks with thousands of resource constrained nodes. The major goal for RPL is to achieve an efficient and reliable routing from any node to the sinks. Although RPL is still an RFC, it has gained much maturity to turning it as a promising standardized routing protocol for LLNs. 
RPL is a distance vector protocol that organizes nodes into a destination oriented directed DAG (DODAG) rooted towards one DAG root (a sink). In RPL, DODAGs are optimized according to an objective function (OF) which can be used to minimize the cost of reaching the root. Objective functions in RPL determine how parent selection and forwarding decisions are made. Considering the large number of applications for RPL, Multiple objective functions exist, each aiming to optimize different metrics [5]. The rank defines individual node positions with respect to the DODAG root. Its exact calculation is left to the objective function, but, to allow loop-detection, rank must monotonically decrease as the DODAG is followed towards the DODAG destination.

\section{B. RPL control messages}

RPL messages are specified as a new type of ICMPv6 control messages. There are three main messages: the DODAG Information Object (DIO), the DODAG Destination Advertisement (DAO), and the DODAG Information Solicitation Message (DIS). Although the RPL is specified by RFC6550 [3], there are still several open research problems need to be addressed. One of the most important issues which still left open is the specification of the Objective Function. In the current RFC specification, there is no clear relation on the rank computation.

\section{PROTOCOL DESIGN}

\section{A. DODAG construction}

Unlike RPL, which is generally a proactive routing protocol where a sink broadcast its presence periodically, this protocol adopts a reactive on-demand approach to adapt rapid changing of the WSN. Every node in the network maintains its own routing table freshness due to the advantage of this approach. Only the sink node (the DODAG root) participates in the process. The DODAG construction is based on the neighbor discovery, which consists of two main operations, (1) broadcasting of a DIS message by a source node to the sink node to request DODAG construction, (2) broadcasting of a DIO message by the sink node to construct a new DODAG.

Before sending a data packet, the source node needs to broadcast a DIS message to a sink node in order to request the DODAG construction. After receiving the DIS message, the sink node increases DODAGID by one as a new DODAG, and initializes rank value to 1 . Then it broadcasts the DIO message onto the network.

Figure 1 shows the process performed by route nodes when they receive a DIO message. When a route node $\mathrm{N}$ receives the DIO message, it compares its DODAGIDs with the DODAGID that is contained in the DIO message. If the received DODAGID is larger than all the DODAGIDs of the node $\mathrm{N}$, which indicates the message of a new DODAG construction, the node $\mathrm{N}$ replaces its DODAGID with received DODAGID and assigns its rank that is equal to received rank incremented by some self-decided value (see details in Subsection III-C).

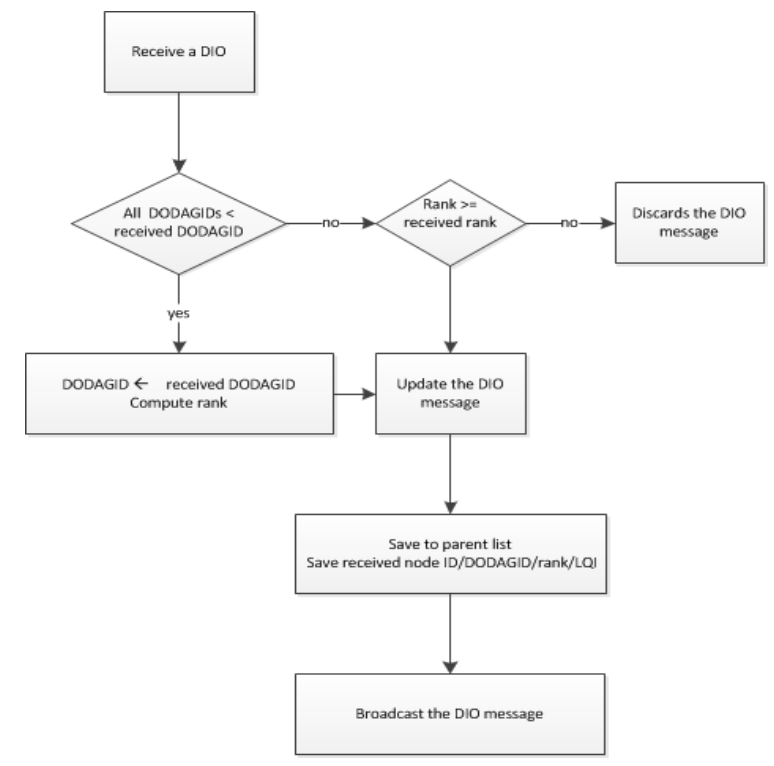

Figure 1. The process performed by route node

If received DODAGID is equal to the node N's DODAGID, the sender can be regarded as another alternate parent node (with higher rank) or a sibling node (with equal rank). Then node $\mathrm{N}$ also updates the DIO message. However, if the received rank is larger than the node N's rank, the node $\mathrm{N}$ discards the DIO message since the sender can be its descendent node in order to avoid loop creation. After having finished updating the DIO message, the node $\mathrm{N}$ creates a new entry in the parent list that contains candidate parents and siblings that can be used if the currently selected parent loses its routing ability. In the construction process of network, the node $\mathrm{N}$ saves the sender' node ID, DODAGID, rank and LQI information to the parent list. Then, it broadcasts the updated DIO message. The constructed DODAG expires after a predetermined period of time.

\section{B. Routing metrics, preferred parent election}

In our implementation, a node selects the neighbor that has the lowest rank value as the preferred parent.

The ROLL group has specified in RFC6551 [6] the Node Energy, Hop Count, Link Throughput, Link Latency, Link Quality Level and Expected Transmission Count routing metrics, while leaving space for defining additional primary routing metrics. As routing metrics, we choose the hop count and the node energy because the path can change rapidly while nodes are moving within the WSNs. The hop count metric outperforms all of the link-quality metrics. Furthermore, the hop count is simple and easy to compute since it requires no additional measurements.

Node energy is a critical metric in WSNs, especially in the presence of battery-operate nodes. If the node energy is not taken into account, then some paths can be more loaded than others and nodes closer to the sink are more subject to premature energy depletion, since they have to relay more packets, which can eventually lead to network disconnection. 
In addition, we uses link quality indication (LQI) [7] to determine priority among the nodes that have the same rank. According to IEEE 802.15 .4 , LQI is characterized by the strength and the quality of a received packet. Several recent researches within the IETF ROLL Working Group recommend the use of LQI.

\section{Rank Computation}

The rank of a node is a scalar representation of the location of the node in a DODAG. Its exact calculation is left to the objective function, but, to allow loop-detection, rank must monotonically decrease as the DODAG is followed towards the DODAG's destination. Each node selects as parent the neighbor which advertises the minimum rank value. It is the Objective Function (OF) that defines how routing metrics are used to compute the rank, and as such it must demonstrate certain properties.

[8] Shows combining hop count with the node energy in a lexical manner, where the hop count is inspected first and only if two paths have equal hop count metric then the node energy metric value is inspected, and the energy consumption of all nodes converges since the forwarding load is distributed among all one-hop neighbors of the destination-sink node. So, in our paper, we combine the hop count metric with the node energy in a lexical manner. In MDMR, the hop count rank can be calculated as

$$
\operatorname{Rank}_{i}=\operatorname{Rank}_{j}+1 \text {, }
$$

Where node $j$ is node $i$ 's potential parent.

The node energy rank can be calculate as

$$
\operatorname{Rank}_{i}=\operatorname{Rank}_{j}+\omega_{i, j}
$$

Where node $j$ is node $i$ 's potential parent and $\omega_{i, j}$ is a function (denoted by $\omega$ in the following) of the characteristics of node $j$ and of the link between nodes $i$ and $j$. This can be expressed as

$$
\begin{gathered}
\omega(\alpha+\beta)=\omega(\alpha)+\omega(\beta), \\
\omega(\alpha)=\frac{V_{\max }^{i}}{V_{\text {now }}^{i}},
\end{gathered}
$$

Where $\alpha$ is the path from the root to node $j, \beta$ is the link between node $i$ and $j$, and $\oplus$ denotes the path concatenation operation. $\mathrm{V}_{\max }$ denotes Maximum (initial) energy and $\mathrm{V}_{\text {now }}$ denotes the current energy value.

\section{DODAG maintenance and repair}

In the protocol, the DAG can provide redundancy path since the node can have multiple parents. After constructing a DODAG, an intermediate node receiving a data packet should search its parent list to determine the next node to forward the data packet. A node with a lower rank and the highest LQI value is chosen as the next node.

Repair mechanisms are key components of routing protocol to dynamically update the routing decisions to adapt the network topology changes. Protocol supports local repair and global repair. When a link or node fails, the node triggers a local repair to quickly find an alternate path without trying to repair the whole DODAG. In addition, the proposed global repair scheme can ensure the recovery of the network even when neither sibling nor parent nodes are accessible by reconstructing DODAG. Therefore, a node can repair broken routes both locally and globally when network topology changes frequently under high mobility scenarios.

\section{SIMULATION ANALYSIS AND RESULTS}

\section{A. Simulation setup}

For the purpose of performance evaluation under more realistic network factors we have carried out simulations. We validate our routing protocol by simulations using ns-2.5. Up to 256 sensor nodes are randomly deployed in a square area $800 \mathrm{~m} \times 800 \mathrm{~m}$. Source nodes are randomly deployed into the sensor field and the mobile speed of sinks varies from $2 \mathrm{~m} / \mathrm{s}$ to $10 \mathrm{~m} / \mathrm{s}$. Radio transmission range of each node is 50 meters. We use 1 Mbps IEEE 802.11b DCF as the MAC protocol, and the energy consumption values are determined according to the study results of Chipcon CC2420 [9]. A traffic generator is developed with 1 query per $1 \mathrm{~s}$ and 1 data report per $0.5 \mathrm{~s}$ during 400 seconds simulation time. The size of data payload is 200 bytes.

We consider two scenarios: (i) scenario 1: the number of sinks is set to 6 and the mobile speed of sinks varies from $4 \mathrm{~m} / \mathrm{s}$ to $20 \mathrm{~m} / \mathrm{s}$, and (ii) scenario 2: the mobile speed of sinks is set to $12 \mathrm{~m} / \mathrm{s}$ and the number of sinks varies from 2 to 10 . We evaluated the performance of MDMR under the network scenario described above and make comparisons with the performance of Directed Diffusion (DD) [10] and TTDD [11]. We use two main metrics to evaluate the performance, namely, energy consumption per node, and average end-toend delay.

\section{B. Simulation results}

In order to examine the impact of sink speed, average energy consumption and average delay are measured for

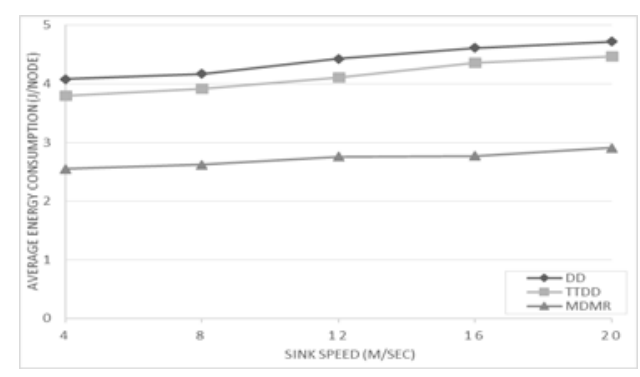

Figure 2. Energy consumption with different sink spped 


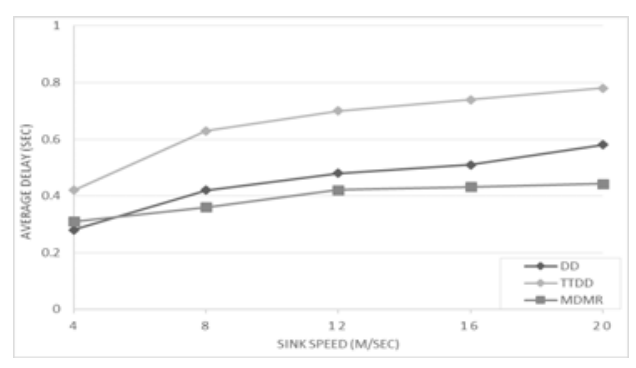

Figure 3. Average dealy with different sink spped

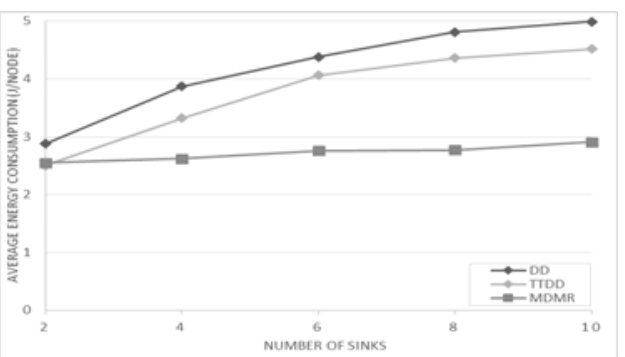

Figure 4. Energy consumption for the number of sinks

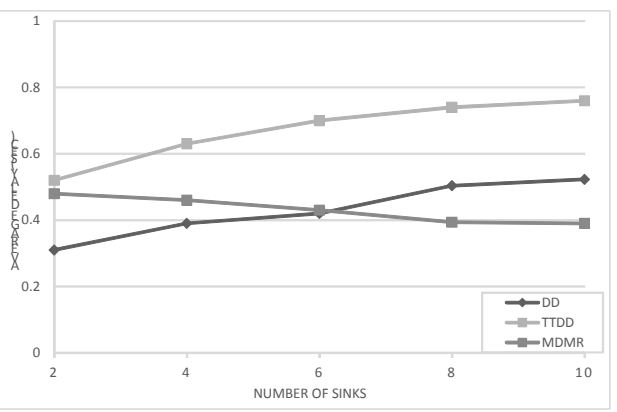

Figure 5. Average dealy for the number of sinks

different sink speeds in a sensor network consisting of 6 mobile sinks and 256 sensor nodes. Figure (2) plots the energy consumption as the sinks' speed changes. Note that MDMR is the most energy efficiency compared to DD and TTDD. This is because that MDMR minimizes control messages exchanges on route failures by using local and global repair mechanisms. Figure (3) shows the the average end-to-end delay increase as the sink speed increase. The slope of the curve decreases with sink speed because a new lower delay path is built more often at higher speeds. In MDMR, local repair mechanism can direct a data message through an alternative path using alternate parents or siblings when a link fails. However, the mechanism does not guarantee the shortest path.

In the following experiments, we investigate the function of the number of mobile sinks. Figure (4) shows energy consumption as the number of sinks is varied, when all sinks move at an average speed of $10 \mathrm{~m} / \mathrm{s}$. In the figure, MDMR demonstrates better energy consumption than the other protocols, which benefits from the repair mechanisms. Furthermore, mobility is handled efficiently without excessive DAG restructuring. In contrast, in DD, the new location of the mobile sink is propagated throughout the sensor field in order for all sensor nodes to get the sink's location. TTDD rebuilds a new multi-hop path between the sink and the grid. Figure (5) shows the average end-to-end delay changes as the number of sinks increases. The average end-to-end delay of MDMR decreases as the number of sinks increases. This effect is the increasing density of sink nodes. In contrast, the average end-to-end delay of DD and TTDD increases due to increasing the tree's depth. The slope of the curve decreases with the number of sinks since there are more chances that a node can exploit an existing path.

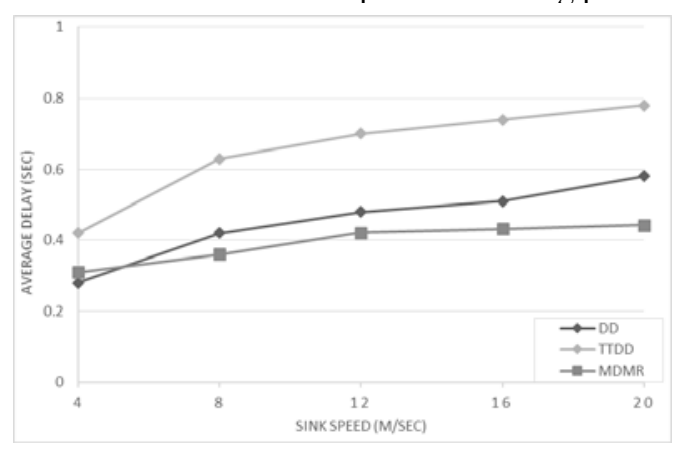

Figure 6. Average dealy with different sink spped

\section{CONCLUSIONS}

In this paper, we propose a multipath routing protocol for DAG-based WSNs with mobile sinks, based on the framework of RPL. MDMR can effectively build the DODAG and provide path redundancy. Furthermore, the local and global mechanisms can ensure data forwarding. Through analytical analysis and realistic simulations, we showed that MDMR outperforms other approaches in DAGbased WSNs with mobile sinks. In the future, we plan to better investigate the routing metrics and the objective function to calculate ranks. These are the key problems to enhance our protocol.

\section{REFERENCES}

[1] Zolt'an Vincze, Rolland Vida, Attila Vid'acs, "Deploying Multiple Sinks in Multi-hop Wireless Sensor Networks", in IEEE International Conference on pervasive Services, July. 2007, pp. 55-63.

[2] Accettura, N. Dipt. di Elettrotec. ed Elettron., Politec., "Performance Analysis of the RPL Routing Protocol”, in RFC 6550, April. 2011, pp 767-772.

[3] T. Winter, Ed., et al., "RPL IPv6 Routing Protocol for Low-Power and Lossy Networks", RFC 6550, IETF, March 2012.

[4] Bogdan Pavković, Fabrice Theoleyre, Andrzej Duda, "Multipath Opportunistic RPL Routing over IEEE 802.15.4", MSWiM '11 Proceedings of the 14th ACM international conference on Modeling, analysis and simulation of wireless and mobile systems, 2011, pp. 1791-186.

[5] P. Thubert, Ed., "Objective Function Zero for the Routing Protocol for Low-Power and Lossy Networks", RFC 6552, IETF, March 2012.

[6] JP. Vasseur, et al., "Routing Metrics Used for Path Calculation in Low-Power and Lossy Networks", RFC 6551, IETF, March. 2012.

[7] C. Gomez, A. Boix, and J. Paradells, "Impact of LQI-Based Routing Metrics on the Performance of a One-to-One Routing Protocol for 
IEEE 802.15.4 Multihop Networks", EURASIP Journal on Wireless Communications and Networking, vol. 2010, 2010, pp. 1-20.

[8] Karkazis, P., Chania, Greece, "Design of primary and composite routing metrics for RPL-compliant WSN", in Telecommunications and Multimedia (TEMU), 2012 International Conference on, Volumn 33, Auguest. 2012, pp. 13-18.

[9] C. -F. Chiasserini and M. Garetto, "An Analytical Model for Wireless Sensor Networks with Sleeping Nodes", IEEE Transactions on Mobile Computing, vol. 5, no. 12, December. 2006, pp. 1706-1718.
[10] C. Intanagonwiwat, R. Govindan, and D.Estrin. "Directed diffusion: A scalable and robust communication paradigm for sensor networks", in Proceedings of the Sixth Annual International Conference on Mobile Computing and Networks, 2000, pp 56-67.

[11] F. Ye, H. Luo, J. Cheng, S. Lu, and L. Zhang. "A two-tier data dissemination model for large-scale wireless sensor networks", in Proceedings of Mobile Computing and Networks, 2002, pp 148-159. 